\title{
A REALTIME EDUCATIONAL CARDIOVASCULAR MODEL OF THE NEONATAL CIRCULATION ON ECMO
}

\section{T. Antonius}

Neonatology, Radboud University Nijmegen Medical Centre, Nijmegen, The Netherlands

\begin{abstract}
A realtime model of the neonatal circulation, oxygen transport and airway mechanics on VA- or VV-ECMO was developed for educational use. The model lets the learner interact with the parameters of the neonatal circulation on ECMO in realtime. In changing and playing with the parameters together with a skilled instructor and ECMO-expert, an invaluable learning experience is created without the need for a real patient. The complex circulation consisting of 87 intertwining variables is made visible through realtime pressure, volume and flow curves which closely resemble a real patient. The model provides the learner with the saturation levels, oxygen content, blood pressure, central venous pressure, cardiac output, pulmonary blood flow and pressure, systemic blood flow and ECMO system pressures in realtime. Neonatal physiological shunts (PDA and OFO) and pathophysiological phenomena (PPHN, sepsis, cardiomyopathy, VSD and congenital defects) are modeled. Learners can rebuild the circulation model using circulation building blocks to construct their own version of the model. Emergencies on ECMO can be simulated (e.g. artifical lung failure, gasflow interruptions, venous return problems) and the influence on the neonatal circulation and oxygenation is shown.
\end{abstract}

The neonatal circulation on ECMO can be very challenging to understand. This model provides insight and let's the learner interact with all the variables of the circulation in order to gain a better understanding of the physiology and pathophysiology of a neonate on ECMO. 